Edmond I Eger II MD

Many of the drugs newly introduced into anaesthetic practice have in common a kinetic profile that permits a more rapid and precise adjustment of effect, including a more rapid recovery of normal function. Sevoflurane and desflurane fit this mold. These new inhaled anaesthetics differ kinetically from isoflurane and halothane because of their lower solubility in blood (Table), a feature produced by halogenation solely with fluorine $\left[\mathrm{CHF}_{2}-\right.$ $\mathrm{O}-\mathrm{CFH}-\mathrm{CF}_{3}$ (desflurane); $\mathrm{CFH}_{2}-\mathrm{O}-\left(\mathrm{CF}_{3}\right)_{2}$ (sevoflurane)]. Desflurane also has lower tissue/blood partition coefficients than any of the other three anaesthetics.

Desflurane differs from isoflurane $\left(\mathrm{CHF}_{2}-\mathrm{O}-\mathrm{CClH}-\right.$ $\mathrm{CF}_{3}$ ) only by the substitution of a fluorine for a chlorine atom. The substitution of fluorine for chlorine changes many properties. It decreases potency (e.g., MAC for sevoflurane in middle-aged patients is $2 \%$ and for desflurane is $6 \%$; compare the latter value with that of $1.15 \%$ for isoflurane) and increases vapour pressure at room temperature $(670 \mathrm{mmHg}$ for desflurane and $170 \mathrm{mmHg}$ for sevoflurane). The MAC-awake (the concentration permitting voluntary response to command in $50 \%$ of patients) is about a third of MAC for both agents. ${ }^{5,6}$ This finding is important because MAC-awake is thought to indicate the concentration providing amnesia in most patients. That is, it suggests that desflurane and sevoflurane are potent amnestics.

The strength of the carbon-fluorine bond increases stability, a fortunate effect because alkali (e.g., soda lime or Baralyme) degrades sevoflurane, especially at the increased temperatures found in the carbon dioxide absorber needed for closed circuit anaesthesia. Were the stability less, sevoflurane might not be clinically useful. In contrast, desflurane resists degradation by alkali and does so more than its chlorinated analog, isoflurane.

The lower solubility of desflurane and sevoflurane indicates a more rapid rate of rise of the alveolar concentration towards the concentration inspired. Results from several studies confirm this prediction. ${ }^{78}$ In the case of sevoflurane, the rapidity of change correctly implies a rapidity of induction of anaesthesia. Sevoflurane has replaced halothane for induction of anaesthesia in children in at least one hospital in Japan, a country where sevoflurane has been released for clinical use. In contrast, the pungency of desflurane results in respiratory tract

\section{New inhalational agents - desflurane and sevoflurane}

irritation and coughing, breathholding and laryngospasm, particularly at concentrations of $7 \%$ or greater. These responses limit desflurane's usefulness as an induction agent, and desflurane is not recommended for this purpose, especially in children.

Once induction is complete, the issue of pungency appears to be minor or non-existent as a factor affecting clinical practice. During maintenance; the concentration of desflurane or sevoflurane can be rapidly adjusted to meet changing clinical needs. Furthermore, the difference between the concentration inspired and that in the alveoli is relatively small. That is, the alveolar concentration can be known with fair precision by knowing the concentration inspired. In turn, given a modest inflow rate (2 $\mathrm{L} \cdot \mathrm{min}^{-1}$ or greater), the difference between the concentration delivered from the vaporizer and that in inspired gas becomes small. Thus, the alveolar concentration and the level of anaesthesia may be controlled and known if one uses an accurately calibrated vaporizer and a modest inflow rate. The same is not true for more soluble anaesthetics where the difference between the concentration delivered and that in the alveoli may be considerable.

As would be predicted from their low solubilities, recovery from anaesthesia during the first 10-20 min after anaesthesia is faster with either agent than with isoflurane. ${ }^{9-11}$ In addition, the more rapid return towards normal from desflurane anaesthesia may be documented for at least an hour and a: half after the termination of anaesthetic administration. ${ }^{10,12}$ Whether this translates into a more rapid release from the recovery room and a more rapid return to normality at home or on the job remains to be adequately tested, but the results of at least one study suggest this possibility for desflurane. ${ }^{12}$ To make use of the potential for an earlier release from the recovery room may require the development of new guidelines for the dismissal of patients.

Many of the remaining pharmacological characteristics of desflurane and sevoflurane resemble those familiar to the practitioner who administers agents such as isoflurane or halothane. All depress respiration, raising $\mathrm{PaCO}_{2}$ and decreasing the ventilatory response to imposed increases

From the Department of Anesthesia, University of California, San Francisco, CA. 
TABLE Human blood/gas and tissue/blood partition coefficients (Mean \pm SD) ${ }^{1-4}$

\begin{tabular}{lcccc}
\hline Tissue & Desflurane & Sevoflurane & Isoflurane & Halothane \\
\hline Blood & $0.42 \pm 0.02$ & $0.69 \pm 0.05$ & $1.46 \pm 0.09$ & $2.54 \pm 0.18$ \\
Brain & $1.3 \pm 0.1$ & $1.7 \pm 0.1$ & $1.6 \pm 0.1$ & $1.9 \pm 0.2$ \\
Heart & $1.3 \pm 0.2$ & $1.8 \pm 0.2$ & $1.6 \pm 0.2$ & $1.8 \pm 0.3$ \\
Liver & $1.3 \pm 0.1$ & $1.8 \pm 0.2$ & $1.8 \pm 0.2$ & $2.1 \pm 0.3$ \\
Kidney & $1.0 \pm 0.1$ & $1.2 \pm 0.2$ & $1.2 \pm 0.2$ & $1.0 \pm 0.2$ \\
Muscle & $2.0 \pm 0.6$ & $3.1 \pm 1.0$ & $2.9 \pm 1.0$ & $3.4 \pm 1.4$ \\
Fat & $27 \pm 3$ & $48 \pm 6$ & $45 \pm 6$ & $51 \pm 10$ \\
\hline
\end{tabular}

in $\mathrm{PaCO}_{2}$. Like isoflurane, desflurane irritates the respiratory tract, whereas sevoflurane (like halothane) does not.

Several cardiovascular effects of desflurane and sevoflurane parallel those of isoflurane and halothane. All decrease blood pressure, but not necessarily by the same mechanism. Desflurane and isoflurane tend to sustain cardiac output, decreasing blood pressure by decreasing systemic vascular resistance. Sevoflurane may share this effect. In contrast, halothane decreases pressure by decreasing cardiac output without affecting systemic vascular resistance. All four agents depress myocardial contractility, halothane causing the greatest depression. Sevoflurane appears to resemble halothane in its effect on heart rate, neither agent increasing rate. Both isoflurane and desflurane increase rate. Desflurane increases rate in a dose-dependent manner, having little effect at light levels of anesthesia (e.g., those near 1 MAC) but causing tachycardia at deep levels. Isoflurane produces a less dose-dependent effect on rate, increasing rate at all levels. The ethers (desflurane, isoflurane, sevoflurane) do not increase sensitivity to the arrhythmogenic effects of exogenously administered epinephrine whereas the alkane (halothane) does predispose to arrhythmias. Coronary steal does not appear to occur with either sevoflurane or desflurane. In at-risk patients, an increase in heart rate and blood pressure may be associated with an increase in myocardial ischaemia during induction of anaesthesia with desflurane (or any agent). In the absence of heart rate and pressure increases, no increase in the incidence of ischaemia has been reported. Desflurane has been used in several hundred patients with coronary artery disease without increasing the incidence of untoward outcomes (e.g., myocardial infarction or death), compared to the incidence with other approaches to anaesthesia.

Both desflurane and sevoflurane depress the electroencephalogram in a dose-related manner, neither causing convulsive activity. Both decrease cerebrovascular resistance and can increase intracranial pressure, and do so in a dose-related manner. These effects resemble those of isoflurane.
Both desflurane and sevoflurane cause muscle relaxation sufficient to permit endotracheal intubation or the conduct of intraabdominal procedures. Both enhance the action of muscle relaxants, a desirable effect because the elimination of the inhaled agents should remove the enhancement and thereby assist in the recovery from paralysis.

Desflurane and sevoflurane differ in their resistance to biodegradation. Desflurane resists biodegradation. The degradation is so small that it is difficult to measure accurately, but it is estimated that only $0.02 \%$ of the desflurane taken up during its administration can be recovered as urinary metabolites. The metabolism of sevoflurane is approximately 100 times greater, slightly exceeding that of enflurane. Inorganic fluoride and trifluoroacetate result from the metabolism of desflurane. Inorganic fluoride and hexafluoroisopopanol result from the metabolism of sevoflurane.

Biodegradation of anaesthetics is of concern because of the known connection between degradation and toxicity for several older anaesthetics such as chloroform, methoxyflurane, halothane, and possibly enflurane. By this reasoning, desflurane should be without toxicity, and the results from studies in animals and approximately 2,000 humans bear out this prediction. Because of its metabolism, sevoflurane is less above suspicion. However, extensive studies in animals and humans have not revealed evidence of toxicity. Sevoflurane has been used in several hundred thousand patients in Japan without reports of a causal association between its administration and hepatic or renal injury.

In summary, both desflurane and sevoflurane offer advantages over other modern inhaled anaesthetics. Both agents provide a greater precision of control over anaesthetic administration, and both permit a significantly more rapid recovery from anaesthesia. An absence of pungency recommends the use of sevoflurane for a rapid induction of anaesthesia by inhalation, whereas desflurane has a pungency that hinders induction by inhalation. Both agents little affect heart rate at light levels of anaesthesia, but desflurane increases rate at deeper levels whereas sevoflurane does not. Desflurane strongly resists 
biodegradation whereas sevoflurane is vulnerable to degradation. Neither agent appears to injure the kidney or liver. Finally, unlike present potent agents, these new anaesthetics can provide anaesthesia in the absence of nitrous oxide without compromising recovery from anaesthesia.

\section{References}

Overviews of the pharmacology of sevoflurane and desflurane are provided in editorials by Jones, ${ }^{13}$ Miller and Green, ${ }^{14}$ and Saidman. ${ }^{15}$

1 Eger EI II. Partition coefficients of 1-653 in human blood, saline, and olive oil. Anesth Analg 1987; 66: 971-3.

2 Yasuda N, Targ AG, Eger EI II. Solubility of I-653, sevoflurane, isoflurane, and halothane in human tissues. Anesth Analg 1989; 69: 370-3.

3 Strum DP, Eger EI II. Partition coefficients for sevoflurane in human blood, saline, and olive oil. Anesth Analg 1987: 66: 654-6.

4 Eger RR, Eger EI II. Effect of temperature and age on the solubility of enflurane, halothane, isoflurane and methoxyflurane in human blood. Anesth Analg 1985; 64: 640-2.

5 Jones RM, Cashman JN, Eger EI II, Damask MC, Johnson $B H$. Kinetics and potency of desflurane (I-653) in volunteers. Anesth Analg 1990; 70: 3-7.

6 Katoh T, Suguro Y, Nakajima R, Kazama T, Ikeda $K$. Blood concentration of sevoflurane and isoflurane on recovery from anaesthesia. Br J Anaesth 1992; 69: 259-62.

7 Yasuda N, Lockhart SH, Eger EI II, et al. Comparison of kinetics of sevoflurane and isoflurane in humans. Anesth Analg 1991; 72: 316-24.

8 Holaday DA, Smith FR. Clinical characteristics and biotransformation of sevoflurane in healthy human volunteers. Anesthesiology 1981: 54: 100-6.

9 Eger EI II, Johnson BH. Rates of awakening from anesthesia with I-653, halothane, isoflurane, and sevoflurane: a test of the effect of anesthetic concentration and duration in rats. Anesth Analg 1987; 66: 977-82.

10 Ghouri $A F$, Bodner $M$, White PF. Recovery profile after desflurane-nitrous oxide versus isoflurane-nitrous oxide in outpatients. Anesthesiology 1991; 74: 419-24.

11 Frink EJ Jr, Malan TP, Atlas M, Dominguez LM, DiNardo $J A$, Brown BR Jr. Clinical comparison of sevoflurane and isoflurane in healthy patients. Anesth Analg 1992; 74: $241-5$.

12 Tsai SK, Lee C, Kwan W-F, Chen B-J. Recovery of cognitive functions after anaesthesia with desflurane or isoflurane and nitrous oxide. Br J Anaesth 1992; 69: 255-8.

13 Jones $R M$. Desflurane and sevoflurane: inhalation anaesthetics for this decade? Br J Anaesth 1990; 65: 527-36.

14 Miller ED, Greene NM. Waking up to desflurane: the anesthetic for the 90s? Anesth Analg 1990; 70: 1-2.
15 Saidman $L J$. The role of desflurane in the practice of anesthesia. Anesthesiology 1991; 74: 399-401. 


\section{Les nouveaux agents inhalatoires: le desflurane et le sévoflurane}

Edmond I. Eger II MD
Plusieurs nouvelles drogues introduites en pratique anesthésique ont un profil cynétique commun qui permet le contrôle plus rapide et plus précis de leurs effets ainsi qu'un retour plus rapide des fonctions normales. Le sévoflurane et le desflurane sont de cette classe. Ces nouveaux agents diffèrent de l'isoflurane et l'halothane sous l'aspect cynétique par leur solubilité sanguine plus basse (Tableau), une charactéristique unique de l'halogénation au fluor $\left[\mathrm{CHF}_{2}-\mathrm{O}-\mathrm{CFH}-\mathrm{CF}_{3}\right.$ (desflurane); $\mathrm{CFH}_{2}-\mathrm{O}-\mathrm{CH}$ $\left(\mathrm{CF}_{3}\right)_{2}$ (sévoflurane)]. Le desflurane a aussi un coefficient de partage tissu/sang plus bas qu'aucun des trois autres anesthésiques.

Le desflurane ne diffère de lisoflurane $\left(\mathrm{CHF}_{2}-\mathrm{O}\right.$ $\mathrm{CClH}-\mathrm{CF}_{3}$ ) par la seule substitution d'un atome de fluor à l'atome de chlore. Cette substitution change plusieurs des propriétés. Elle diminue la puissance (e.g., la MAC du sévoflurane chez le patient d'âge moyen est $2 \%$ et celle du desflurane $6 \%$ comparativement à celle de l'isoflurane qui est $1,15 \%$ ), et augmente la pression de vapeur à la température de la pièce $(670 \mathrm{mmHg}$ pour le desflurane et $170 \mathrm{mmHg}$ pour le sévoflurane). La MAC éveillée (la concentration permettant une réponse volontaire à un ordre verbal dans $50 \%$ des patients) est environ un tiers de la MAC pour les deux agents. ${ }^{5,6}$ Cette donnée est importante parce que la MAC éveillée indique, semble-t-il, la concentration nécessaire pour procurer l'amnésie à la plupart des patients. Ceci suggère donc que le desflurane et le sévoflurane seraient de puissants amnestiques.

La force de la liaison carbone-fluor augmente la stabilité. Cet effet est favorable parce que les alcali (chaux sodée ou Baralyme) dégradent le sévoflurane, particulièrement à la température de l'absorbeur de gaz carbonique générée pour l'anesthésie en circuit fermé. Avec une stabilité moindre, le sévoflurane ne pourrait être utilisé en clinique. Par contre, le desflurane résiste à la dégradation par les alcalis encore mieux que son analogue chloré l'isoflurane.

La solubilité moindre du desflurane et du sévoflurane laisse prévoir une vitesse d'accroissement plus rapide de la concentration alvéolaire vers l'équilibre avec la concentration inspirée. Les résultats de plusieurs études confir- ment cette prédiction. Dans le cas du sévoflurane, la rapidité du changement de concentration corrélère bien la rapidité d'induction de l'anesthésie. Le sévoflurane a remplacé l'halothane comme agent d'induction chez les enfants dans au moins un hôpital au Japon, pays ou il est maintenant disponible pour usage clinique. Par contre, l'odeur âcre du desflurane irrite les voies respiratoires. Elle provoque de la toux, de la retenue respiratoire et du laryngospasme, particulièrement avec des concentrations plus élevées que $7 \%$. Ces inconvénients limitent l'utilisation du desflurane comme agent d'induction. Cet agent ne peut donc être recommandé dans ce but, surtout chez les enfants.

Une fois l'induction réalisée, le problème de l'âcreté comme facteur défavorable disparaît à toutes fins pratiques. Pendant le maintien, la concentration du desflurane et du sévoflurane peut se contrôler facilement pour atteindre les effets cliniques désirés. De plus, la différence entre la concentration inspirée et celle de l'alvéole est relativement faible. Ainsi, on connaît la concentration alvéolaire assez exactement par la concentration inspirée. Avec un débit d'arrivée modeste $\left(2 \mathrm{~L} \cdot \mathrm{min}^{-1}\right)$, la différence entre la concentration transmise par le vaporisateur et celle du gaz inspiré devient elle aussi minime. La concentration alvéolaire et la profondeur de l'anesthésie peuvent être connues et ajustées facilement si on utilise un vaporisateur calibré avec précision et un débit d'arrivée modeste. Ce qui n'est pas vrai pour les anesthésiques plus solubles pour lesquels la différence entre concentration délivrée et concentration alvéolaire peut être considérable.

Comme on pouvait le prédire par leur solubilité, le réveil qui survient dans les 10-20 min après l'arrêt de l'anesthésie est plus rapide avec ces deux agents qu'avec l'isoflurane. ${ }^{9-11}$ Un retour plus rapide à l'état normal est documenté sur une période d'une heure et demie après l'arrêt de l'anesthésie au desflurane. ${ }^{10,12}$ Il reste à étudier si cette caractéristique se traduit par une sortie plus rapide de la salle de réveil et un retour à la normalité à la maison ou au travail. Dans le cas du desflurane au moins une étude le laisse croire. ${ }^{12}$ Pour réellement utiliser le potentiel d'une sortie plus rapide de la salle de réveil, de nouvelles 
TABLEAU Coefficients de partage sang humain/gaz et Tissu/Sang (Moyenne \pm ET) ${ }^{1-4}$

\begin{tabular}{lcccc}
\hline Tissu & Desflurane & Sévoflurane & Isoflurane & Halothane \\
\hline Sang & $0,42 \pm 0,02$ & $0,69 \pm 0,05$ & $1,46 \pm 0,09$ & $2,54 \pm 0,18$ \\
Cerveau & $1,3 \pm 0$, & $1,7 \pm 0,11$ & $1,6 \pm 0,1$ & $1,9 \pm 0,2$ \\
Coeur & $1,3 \pm 0,2$ & $1,8 \pm 0,2$ & $1,6 \pm 0,2$ & $1,8 \pm 0,3$ \\
Foie & $1,3 \pm 0,1$ & $1,8 \pm 0,2$ & $1,8 \pm 0,2$ & $2,1 \pm 2,3$ \\
Rein & $1,0 \pm 0,1$ & $1,2 \pm 0,2$ & $1,2 \pm 0,2$ & $1,0 \pm 0,2$ \\
Muscle & $2,0 \pm 0,6$ & $3,1 \pm 1,0$ & $2,9 \pm 1,0$ & $3,4 \pm 1,4$ \\
Graisse & $27 \pm 3$ & $48 \pm 6$ & $46 \pm 6$ & $51 \pm 10$ \\
\hline
\end{tabular}

recommandations sur le congé des patients pourraient s’avérer nécessaires.

Plusieurs des autres caractéristiques du desflurane et du sévoflurane ressemblent à celles qui sont familières au practicien qui administre lisoflurane et l'halothane. Ils dépriment tous la respiration, ce qui se traduit par une augmentation de la $\mathrm{PaCO}_{2}$ et une diminution de la réponse ventilatoire aux augmentations de $\mathrm{PaCO}_{2}$. Comme l'isoflurane, le desflurane est irritant pour les voies respiratoires alors que le sévoflurane, comme l'halothane, ne l'est pas.

Plusieurs des effets cardio-vasculaires vont de pair avec ceux de lisoflurane et de l'halothane. Par des mécanismes quelquefois différents, ils abaissent tous la pression artérielle.

Le desflurane et l'isoflurane maintiennent le débit cardiaque mais diminuent la pression artérielle par une baisse de la résistance vasculaire périphérique. Le sévoflurane pourrait agir de cette façon. Par contre, l'halothane diminue le débit cardiaque sans affecter la résistance périphérique. Les quatre agents, l'halothane à un degré plus grand, diminuent la contractilité myocardique. Le sévoflurane se rapproche de l'halothane pour ses effets sur la fréquence cardiaque: aucun des deux agents ne l'augmente. L'isoflurane et le desflurane augmentent la fréquence. L'augmentation causée par le desflurane dépend de la concentration. Cet agent n'a pas beaucoup d'effets à un niveau léger d'anesthésie (près de MAC 1) mais provoque de la tachycardie aux niveaux profonds. L'effet de l'isoflurane sur la fréquence dépend moins de la concentration: elle l'augmente à tous les niveaux d'anesthésie. Les éthers (desflurane, isoflurane, sévoflurane) n'augmentent pas la sensibilité du coeur aux effets arythmogènes de l'épinéphrine exogène alors que l'alcane (halothane) prédispose aux arythmies. Il ne semble pas que le vol coronaire survienne avec le desflurane et le sévoflurane. Chez les patients à risque, l'augmentation de la fréquence cardiaque et de la pression artérielle peut s'accompagner d'une aggravation de l'schémie myocardique pendant linduction avec le desflurane (ou tout autre agent). En l'absence de tachycardie et d'hypertension, on ne rapporte pas d'augmentation de l'incidence de l'ischémie avec le desflurane. On a utilisé le desflurane chez plusieurs centaines de coronariens sans augmenter les conséquences fâcheuses (infarctus du myocarde ou décès) comparativement aux autres agents.

Sans provoquer de convulsions, le desflurane et le sévoflurane dépriment l'électroencéphalogramme proportionnellement à la concentration. Mais comme les deux diminuent la résistance vasculaire périphérique de façon proportionnelle à leur concentration, ils peuvent aussi augmenter la pression intracrânienne de la même façon. Ces effets ressemblent à ceux de lisoflurane.

Les deux agents, desflurane et sévoflurane, relâchent suffisamment la musculature pour permettre lintubation endotrachéale et la chirurgie intra-abdominale. Ils potentialisent l'action des relaxants musculaires. Cet effet est désirable car l'élimination de l'agent facilite la récupération de la force musculaire.

Les deux agents sont différents sous l'aspect de la biodégradation. Le desflurane est résistant. Cette dégradation est si faible qu'on peut à peine la mesurer. On estime que seulement $0,02 \%$ du desflurane capté pendant son administration peut se retrouver sous la forme de métabolites urinaires. Le sévoflurane a un métabolisme 100 fois plus élevé, dépassant même légèrement celui de l'enflurane. Le fluorure inorganique et le trifluoroacétate sont des métabolites du desflurane, alors que le fluorure inorganique et l'héxafluoroisopropanol sont des métabolites du sévoflurane.

La relation qui existe entre la biodégradation des anesthésiques et la toxicité est un sujet d'inquiétude. On lui attribue la toxicité du chloroforme, du méthoxyflurane, de l'halothane et de l'enflurane. Sous cet aspect, le desflurane ne devrait pas être toxique. Les études chez l'animal et chez près de 2000 humains confirment cette prédiction. A cause de son métabolisme, le sévoflurane ne devrait pas échapper aux soupçons. Cependant, des études approfondies chez l'animal et chez l'homme ne révèlent pas de toxicité. Le sévoflurane a été utilisé chez 
des centaines de milliers de patients au Japon sans qu'on rapporte une relation de cause à effet entre son administration et le dommage hépatique ou rénal.

En résumé, le desflurane et le sévoflurane ont des avantages sur les autres agents modernes. Ils peuvent être administrés avec une plus grande précision et ils procurent un réveil plus rapide. L'absence d'âcreté du sévoflurane en fait un agent de choix pour l'induction inhalatoire rapide, ce qui n'est pas le cas du desflurane. En anesthésie légère, ces deux agents ont peu d'effets sur la fréquence cardiaque. Le desflurane augmente la fréquence en anesthésie profonde alors que le sévoflurane ne l'augmente pas. Le desflurane est très résistant à la biodégradation alors que le sévoflurane lui est vulnérable. Aucun de ces agents ne semblent endommager le rein ou le foie. Finalement, contrairement aux autres agents en usage actuellement, ces nouveaux anesthésiques offrent l'avantage d'un réveil rapide même lorsqu'utilisés seuls sans addition de protoxyde d'azote.

\section{Références}

(Voir page R5) 